\title{
Voltage Regulation through Wide Area Voltage Control of HV/MV Transformers and Inverters' Reactive Power Support
}

\author{
I. Talavera ${ }^{1}$, P. Franz ${ }^{1}$, T. Theisen ${ }^{2}$ and J. Hanson ${ }^{1}$ \\ ${ }^{1}$ Department of Electrical Power Supply with Integration of Renewable Energies \\ Technische Universität Darmstadt \\ Landgraf-Georg-Straße 4, 64283 Darmstadt (Germany) \\ Phone number: +496151166587, e-mail: ignacio.talavera@e5.tu-darmstadt.de \\ ${ }^{2}$ Department of New Technologies \\ RWE Deutschland AG \\ Kruppstraße 5, 45128 Essen (Germany) \\ Phone: +49 201 12-29287, e-mail: thomas.theisen@ rwe.com
}

\begin{abstract}
This paper presents a technical evaluation of two different methods to avoid the excessive voltage deviations caused by dispersed generation in distribution grids. Two voltage regulation methods, wide area voltage control of HV/MV transformer and inverters' reactive power support, are simulated in several real rural grids with different topologies located in Northern Germany. In all these analysed grids the high penetration of dispersed generation produces big power flow reversals during low load periods. The objective of implementing both voltage regulation methods is to reduce voltage deviations in such an extent that voltage band violations no longer act as the principal obstacle to host more dispersed generation in the distribution grid. We evaluate the effectiveness of these two methods through a power system analysis program and analyse the reciprocal effects caused by simultaneous use of both methods. Moreover, the concept of "voltage inhomogeneity" is introduced.
\end{abstract}

\section{Key words}

Voltage regulation, dispersed generation, wide area voltage control, reactive power support, voltage inhomogeneity.

\section{Introduction}

The ambitious environmental policy adopted by the German government [1] regarding the energy turnaround has led to a massive penetration of renewable resources in the electrical power system. Unlike conventional power plants, this continuously increasing amount of renewable energy is generated to a high extent by medium or small generators dispersedly located and mainly connected to the lower voltage levels of the power grid.

This new type of generation commonly called Dispersed Generation (DG) is causing serious effects on the distribution grid. The most notable of these effects is the violation of the $10 \%$ voltage limit for end consumers [2].
These voltage violations usually appear well before the thermal limit of the equipment is exceeded. This has the consequence that currently the compliance with voltage limits is acting as the principal obstacle to host more DG and making the present voltage regulation no longer valid. This effect is especially critical in rural areas where the demanded energy density, which was formerly the basis for the layout of the network, is low and MV-and LV-lines are notably long.

Due to the clear trend of increasing the installed DG, it is expected that this voltage situation is reached in many more networks within the following years. These voltage problems could be solved through traditional grid expansion. However, according to some evaluations this would represent in Germany until 2030 around 27 billion euros of investments only for the distribution grid [3]. German Distribution System Operators (DSOs) are therefore looking for alternative solutions that reduce part of these investments and achieve a more economical solution to upgrade the network for the increasing generation.

Two of the possible alternative solutions to enhance voltage profiles are Wide Area Voltage Control of HV/MV Transformer (WAVC-T) and Inverters' Reactive Power Support (IRPS). This paper is focused to analyse the effects of both methods in several rural MV-grids with high penetration of renewable energies and different topologies.

\section{The limits of traditional voltage regulation}

In those distribution grids, where the amount of DG is not sufficient to cause a power flow reversal, the main task for the voltage regulation is to ensure that the maximum negative voltage deviation is not exceeded 
during loading. According to EN 50160 [2], this maximum negative deviation is $-10 \% U_{\mathrm{n}}$.

In this situation the only worst-case regarding the voltage level is peak load. During this situation, voltages at the different nodes reach their lowest values due to the high voltage decreases along the lines and transformers.

In order to avoid the violation of the maximum voltage deviation during peak load, HV/MV transformers are normally equipped with On-Load Tap-Changers (OLTC). This device is automatically controlled and permits to keep the voltage at the MV-busbar within a narrow voltage band independently on loading. This voltage fixed value of the MV-busbar, known as the reference voltage, is adjusted to a higher value than the nominal voltage. Besides permitting a higher voltage decrease without reaching the maximum negative voltage deviation, higher voltages reduce grid losses and decrease operating costs for DSOs.

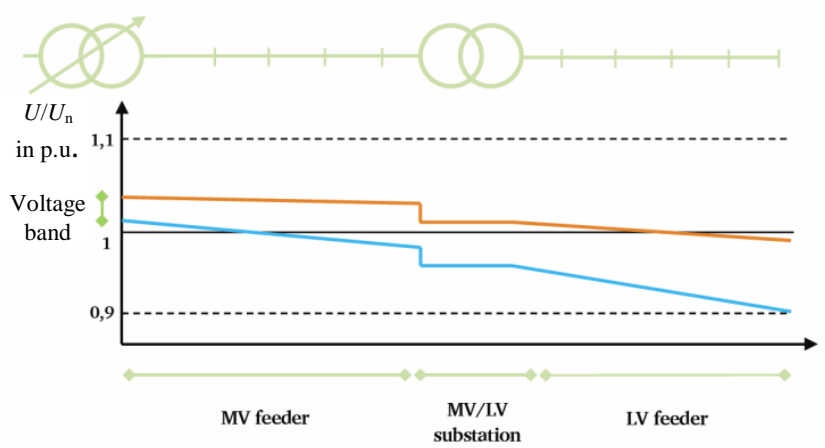

Figure 1: Voltage profile along a distribution feeder during offpeak load (orange) and peak load (blue).

The classical voltage regulation in the distribution grid is based on the "fit and forget" principle. For a fault-free operation this guarantees that these grids respect maximum voltage limits for all possible demand levels without any direct intervention of the DSO.

If distribution grids are characterized by low maximum negative voltage deviations, a downward adjustment of the reference voltage can be implemented and the "fit and forget" principle is further extended even if moderate power flow reversals appear. In this case, the capacity for hosting more DG is easily increased.



Figure 2: Voltage profile along a distribution feeder during maximum reverse power flow (orange) and peak load without feed-ins (blue).
Nevertheless, above a certain amount of DG the calculation of constant reference voltage suitable for both maximum-consumption-no-generation and minimumconsumption-maximum-generation is not possible any more. This occurs when at any node grid the sum of the voltage rise during the highest power flow reversal, the voltage fall during peak load and the regulation bandwidth of the HV/MV transformer exceeds $20 \% U_{\mathrm{n}}$. In this case, there is no possible constant reference voltage that is adequate for the two extreme situations.

\section{Studied methods to upgrade voltage regulation}

\section{A. Wide Area Voltage Control of HV/MV Transformer (WAVC-T)}

To date, the modification of the transformation ratio of HV/MV transformers equipped with OLTC has virtually been the only method to regulate voltages in normal distribution grids. It has to be remarked that this regulation has to be capable of controlling the MV grid and indirectly the LV grid for all the different expected load levels ${ }^{1}$.

The standard operation of these transformers can be explained as follows. A reference voltage for the voltage of the secondary side of transformer is fixed ${ }^{2}$. Voltage is measured at the transformer's secondary side and it is compared with the reference value. When the deviation between the real and the reference voltage can be reduced by a modification of the tap setting, the controller sends an order to actuate to the OLTC.

Up to now, the results of this simple regulation have been satisfactory despite the constant reference voltage. However, voltages at the end of the lines are higher than at the HV/MV-substation when generation exceeds consumption. During this situation a reference voltage higher than the nominal voltage would not be appropriate since the margin for positive deviations is reduced and so the amount of maximum hosted DG. In this context, one logical approach would be to reduce the reference voltage during periods of higher generation than consumption and this is exactly what WAVC-T strives for.

In contrast to the standard control method based on a fixed voltage at the busbar, WAVC-T monitors the grid state by measuring voltages at other nodes apart from the substation and by doing so the reference voltage can be adjusted to the requirements of the "voltage-critical" nodes. As this technology enables a better control of the voltage deviation in MV-grids, it could also represent an indirect help for the voltage regulation in the LV-grid ${ }^{3}$.

\footnotetext{
${ }^{1}$ Typical commercial transformers can be regulated within a range of $\pm 17 \% U_{\mathrm{n}}$ with regulation steps of $1-2 \%$ [4].

${ }^{2}$ This value can be modified for the day, the night, the weekend or different seasons depending on the foreseeable general load levels but it usually remains constant unless the maximum expected peak load is significantly increased.

3 This control is therefore based on a higher degree of observability of the MV-grid.
} 

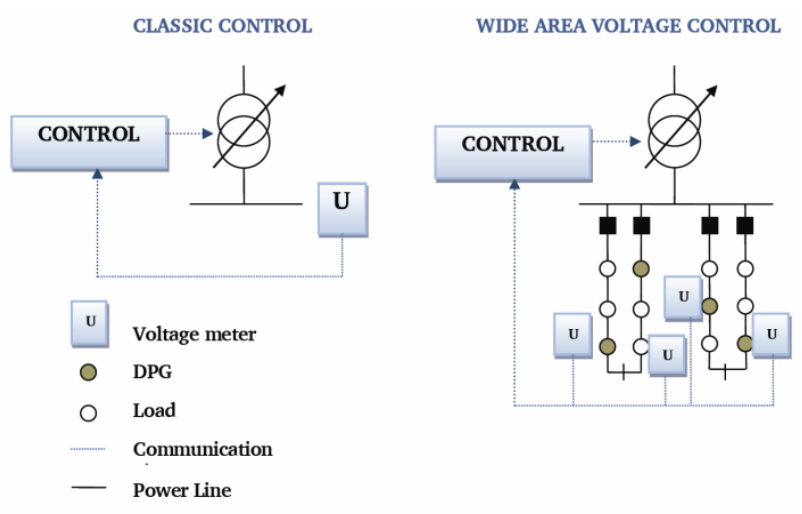

Figure 3: Schematic representation of the operation of the conventional control method and WAVC-T [5].

One characteristic of this technology is its intrinsic slowness and step-like response. Due to these dynamic characteristics this technology is well suited to work with other technological solutions that allow a fast and continuous influence on voltage, for example, IRPS.

The fundamental advantage of this regulation system is the current availability of the fundamental actuator, the tap changer. The global cost of the installation consists of a more sophisticated control system, not very costly, and new measuring devices with their respective communication systems that have to be available at all times, the major part of the costs.

From a technical point of view, the biggest disadvantage of this system is however based on its one degree of freedom. At any time tap position has to be adequate for every node of the grid. Consequently, this regulation may not be capable of maintaining the voltage within the stipulated limits in those distribution grids whose lines are long and have different ratios load to generation. Since the MV-grid can cover considerable areas, feed several lines and its dimensioning was based on energy consumption and not on possible energy feed-in, this ratio differs substantially from one feeder to the other.

From now on, those grids whose difference between the maximum and minimum voltage is sufficient to obstacle the operation of WAVC-T will be described as "voltageinhomogeneous".

For a comprehensive evaluation of WAVC at least two other aspects have to be analysed: grid losses and switching rate of OLTC. The former has a direct impact on the DSO's costs and the latter on the lifetime of OLTC.

\section{B. Inverters' Reactive Power Support (IRPS)}

One possible solution to minimise voltage deviations caused by DG is to accompany this active power feed-in with a certain amount of reactive power absorption. In accordance with the present German grid code requirements, generation units with a certain rated power have to be able to modify their power factor until 0,9 or 0,95 for the LV-and MV-grid, respectively [6] [7].

To comply with the commented guidelines, the compulsory behaviour of DG regarding reactive power support can be sorted out as follows:

1) Constant power factor $\cos \varphi$

2) Variable power factor dependent on active power generation $\cos \varphi(P)$

3) Constant reactive power supply $Q$ in $\mathrm{VAr}$

4) Reactive Power/Voltage characteristic curve $Q(U)$

In the LV-grid the most common set value used by DSO are $\cos \varphi(P)$ curves. Figure 4 shows an example of a commonly used curve. In this case, the power factor is set at one until half of the rated power is reached. Since voltage problems normally do not appear for low generations, additional losses caused by reactive power can be avoided. This power factor is linearly reduced for power values higher than half the nominal power until it reaches either 0,9 or 0,95 (inductive) for a power value close to its nominal power.



Figure 4: Example of a commonly used $\cos \varphi(P)$ curve for IRPS [8].

Independently of how it is carried out, IRPS has some advantages that make it very convenient for a wide spread use in the distribution grid. In case of implementing constant characteristic curves no additional equipment needs to be installed by the DSOs and, thus, no direct investments are required. Moreover, the legal framework clearly permits DSOs, at least in Germany, to demand DG to implement a control of their reactive power supply.

However, there are two main drawbacks for the implementation of IRPS. The higher reactive power flows cause additional losses. The extra losses rise the operating costs of the DSO. Furthermore, the additional reactive power flow increases the apparent power through the grid and can bring the electrical equipment to its thermal limit if it was initially highly loaded.

\section{Assumptions and scenarios for the simulations}

We will analyse the behaviour, effects and potential use of WAVC-T and IRPS for voltage regulation in several rural MV-grids. The LV-grids connected to the MV-grids will only be modelled as PQ-loads with a power factor of 0,95 inductive. LV-connected DG will be modelled as if 
they feed directly energy at the MV node without considering the LV-feeder and local transformer.

For the maximum voltage deviation we take as basis the European standard for power quality in LV-grids [2]. Since the LV-grids connected to the studied MV-grids will not be directly analysed, a maximum deviation for the $\mathrm{MV}$-grids has to be defined. For these analyses, we assume maximum voltage deviations of $4 \% U_{\mathrm{n}}$ at the MV-nodes. Based on [2], this value allows a further voltage deviation increase of $2 \% U_{\mathrm{n}}$ along the MV/LV substations and of $4 \%$ $U_{\mathrm{n}}$ along the LV-feeders [3].

To implement WAVC-T we assumed that both the minimum and the maximum voltage of the grid are inside the voltage measurements received by the control unit. The control unit calculates an appropriate tap setting that keeps the voltage level between $\pm 4 \% U_{\mathrm{n}}$ at all nodes. If this were no possible, the tap setting that causes the minimum voltage deviation would be selected.

The implementation of IRPS consists of the $\cos \varphi(P)$-curve described in Figure 4. During maximum generation, DG will operate at 0,95 underexcited regardless their nominal power and location.

Since the grid is designed to operate adequately during the traditional worst-case and consumption has not significantly changed ${ }^{4}$, the scenarios to investigate voltage deviations are the following:

1) Minimum-consumption-maximum-generation

2) Maximum-consumption-maximum-generation

Whereas the first scenario simulates the highest voltages and therefore the highest positive voltage deviations, the second scenario is defined to analyse voltage differences among feeders. As already commented, these could hinder the effective use of WAVC-T.

The simulation of these scenarios is based on scaling factors used to adjust nominal powers of loads and DPGs. The combination of the scaling factors simulates the above mentioned load-flow scenarios:

\begin{tabular}{|c|c|c|}
\hline & $\begin{array}{c}\text { Minimum } \\
\text { Consumption } \\
\text { Maximum } \\
\text { Generation }\end{array}$ & $\begin{array}{c}\text { Maximum } \\
\text { Consumption } \\
\text { Maximum } \\
\text { Generation }\end{array}$ \\
\hline Load & 0,4 & 1 \\
\hline PV-units & 0,8 & 0,8 \\
\hline Other DPGs & 1 & 1 \\
\hline
\end{tabular}

\section{Simulation results}

\section{A. Initial situation}

The results of the simulations show that during a scenario of minimum-consumption-maximum-generation voltage

\footnotetext{
${ }^{4}$ Maximum-consumption-no-generation.
}

deviations exceed $8 \% U_{\mathrm{n}}$ in all analysed MV-grids. This is more than twice the maximum fixed voltage deviation for MV-grids.

It has to be remarked that despite the large violation of the voltage limits no grid element is thermally overloaded in any MV-grid and scenario. These results highlight that voltage limits are the main burden to host large amounts of DG in the distribution grid.

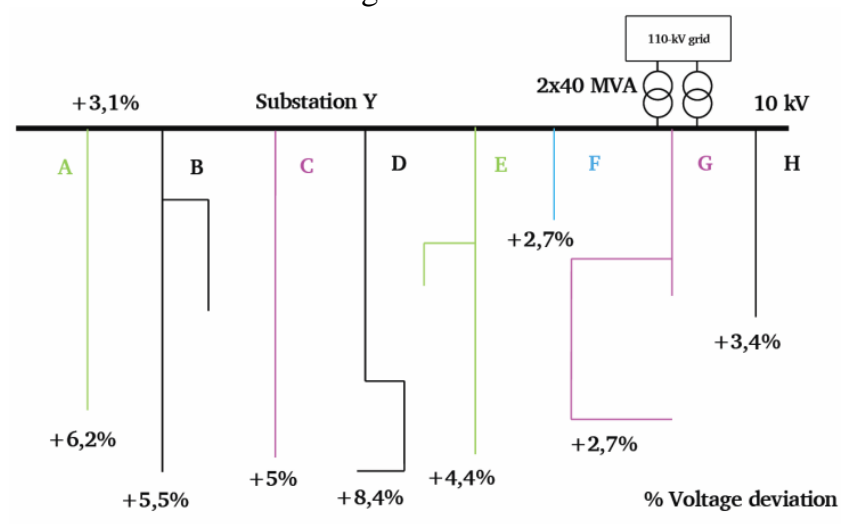

Figure 5: Maximum voltage deviations in grid "Y" during minimum-consumption-maximum-generation.

\section{B. Wide Area Voltage Control of HV/MV Transformer (WAVC-T)}

WAVC-T is able of improving voltage deviations considerably for all the grids and scenarios. This is especially the case for those grids whose feeders do not have extremely different ratios of generation to consumption.

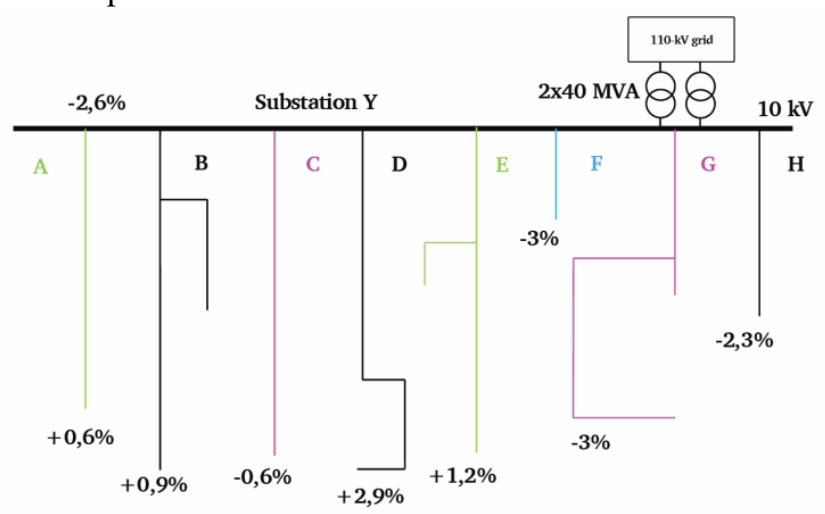

Figure 6: Maximum voltage deviations in grid "Y" during minimum-consumption-maximum-generation with WAVC-T.

In one of the analysed MV-grids, grid "Z", we observe that maximum voltage deviation cannot be held within $\pm 4 \% U_{\mathrm{n}}$ for a scenario of maximum-consumptionmaximum-generation.

Grid " $\mathrm{Z}$ " shown in Figure 7a is characterised by two long MV feeders, "E" and "G". During the mentioned scenario, a strong power flow reversal appears in feeder "E" whereas load largely exceeds generation in feeder "G". Therefore, voltage deviations are considerable in both feeders but with opposite sign. As already explained, this grid can be described as voltage inhomogeneous. 
Figure $7 \mathrm{a}$ and Figure $7 \mathrm{~b}$ show the HV/MV transformer is not able to set a tap position where both the maximum positive and maximum negative deviations are simultaneously held within $\pm 4 \% U_{\mathrm{n}}$.

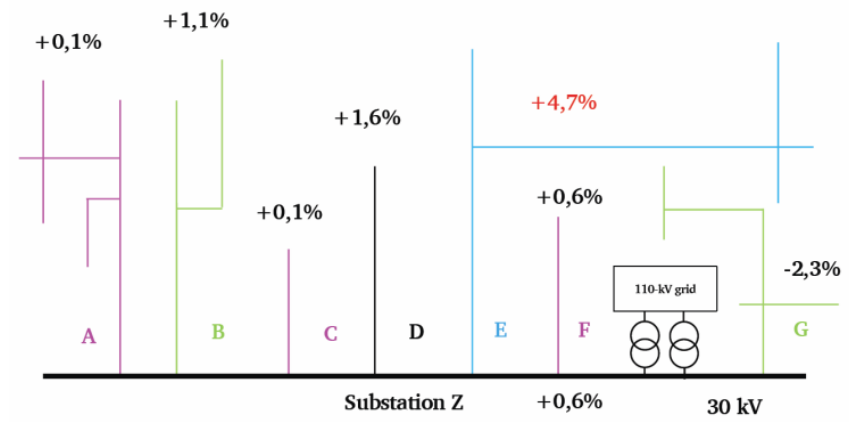

Figure 7a: Maximum voltage deviations in grid " $Z$ " during maximum-consumption-maximum-generation with WAVC-T. High tap setting.

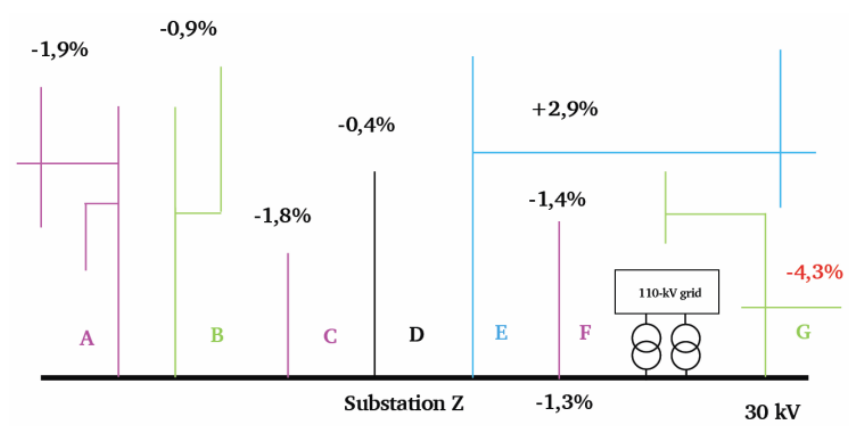

Figure 7b: Maximum voltage deviations in grid " $\mathrm{Z}$ " during maximum-consumption-maximum-generation with WAVC-T. Low tap setting.

Although the violation of the voltage deviation for the present situation is minimal, this effect could worsen. For example, if an increase of the maximum generation in "E" takes place in the next years.

\section{Inverters' Reactive Power Support (IRPS)}

In all the analysed MV-grids, IRPS achieves a significant reduction of the maximum voltage. Additionally, a further decrease of voltage should also be expected along those $\mathrm{MV}$-grids where DG is connected to.



Figure 8: Maximum voltage deviations in grid "Y" during minimum-consumption-maximum-generation with IRPS.

The voltage reduction in an investigated MV-grid can be seen by a comparison of Figure 5 to Figure 8 . Nevertheless, the target of limiting voltage deviation in
MV-grids to $\pm 4 \% U_{\mathrm{n}}$ is not accomplished in any of the grids.

It has to be remarked that the effectiveness of IRPS depends considerably on the location of the node. Those nodes placed at the end of a $\mathrm{MV}$-feeder receive the accumulated effect of the reactive power flow along the whole line. For those nodes the reduction of the voltage deviation reaches even $2 \% U_{\mathrm{n}}$. The opposite happens for those nodes located close to the HV/MV substation. In this case, voltage deviations are not significantly varied.

This inhomogeneous voltage variation differs from the uniform regulation of the WAVC-T. Whereas the effect of the former is an alteration of the voltage difference between the bus bar and the feeder end, the latter modifies the voltage level in the whole grid homogeneously.

Moreover, another aspect of IRPS is the growth of losses. Due to the bigger reactive power flow, losses are higher than after the implementation of WAVC-T. The increase in power losses with respect to the initial situation is between $14 \%$ and $22 \%$ depending on the grid. However, the effect on the overall yearly energy losses is considerably smaller since on-roof PV-units only operate close to its rated power for very short periods of time and therefore the additional reactive power is very limited in time.

\section{WAVC-T and IRPS}

One notable outcome of the simulations is based on the fact that the WAVC-T combined with IRPS is able to limit voltage deviations successfully in all the analysed MV-grids for all the studied combinations of load and generation. This result is remarkable since due to their characteristics some of the studied grids could be considered one of the most critical examples for voltage regulation in MV-grids.

Furthermore, this combination permits a higher voltage reduction at the end of the lines compared to the implementation of WAVC-T alone. This is based on the fact that in addition to the general lower voltage level achieved by the WAVC-T voltage deviations between the busbar and the end of lines are also reduced in those feeders with high voltages. As it was explained before, this second effect is caused by IRPS.

In the analysed grids we observe that IRPS achieves a reduction of difference between the highest and lowest voltage in the grid. In other words, an improvement of the already defined "voltage inhomogeneity". Since this is the main problem for the adequate operation of WAVC-T, the combination of both methods can be considered to be synergic.

The observed reduction of voltage inhomogeneity in the investigated grids during maximum generation always follows the same pattern: 
1) The highest voltage of the grid appears at the end of a long feeder with a high ratio of generation to consumption. Due to the length of the feeder and the high amount of additional reactive power absorption, proportional to the amount of DG, the decrease of the highest voltage is high.

2) The lowest voltage of the grid appears at the end of a feeder with high consumption and low penetration of DG. Due to this low generation, the amount of additional reactive power caused by the IRPS is small and so is the voltage reduction at the feeder end.

3) Since the highest voltage is more reduced than the lowest voltage, the voltage inhomogeneity decreases.

The decrease of voltage inhomogeneity can be clearly observed by a comparison of Figure 7 to Figure 9. Whereas the difference between the highest and lowest voltage exceeds $7 \% U_{\mathrm{n}}$ in the former figure, the voltage difference does not reach $6 \% U_{\mathrm{n}}$ in the latter. As a result, a suitable tap position can be found in grid " $Z$ " for maximum-consumption-maximum-generation.

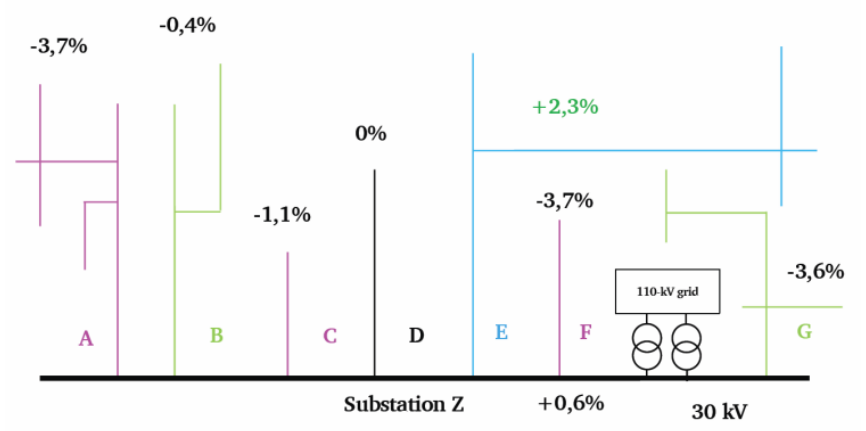

Figure 9: Maximum voltage deviations in grid " $Z$ " during maximum-consumption-maximum-generation with WAVC-T and IRPS. Higher tap setting.

In spite of the fact that no one of the analysed grids presents this behaviour, it may be theoretically possible to find grids whose voltage inhomogeneity increases after the implementation of IRPS. An example of this could happen if one feeder had simultaneously the highest amount of DG and a high consumption. If the ratio consumption to generation and the length of the feeder were sufficient high, the voltage in this feeder could reach the lowest value of the grid. For a feeder with these characteristics, the voltage decrease due to IRPS could be also higher than the decrease of the highest voltage. As a result, the voltage inhomogeneity will worsen.

Based on traditional grid planning the probability of finding a feeder with these characteristics is expected to be very low. However, further investigations with a higher amount of real distribution grids are necessary to reach a firm conclusion of the quantitative and qualitative effect of IRPS on the voltage inhomogeneity in MV-grids.

\section{Conclusion}

The first conclusion that can be drawn from the results of the simulations is that the implementation of Wide Area Voltage Control of the HV/MV-transformer (WAVC-T) represents an effective method to reduce voltage deviations. In half of the studied grids WAVC-T is able to limit voltage deviations well below $\pm 4 \% \quad U_{\mathrm{n}}$ for different combinations of consumption and generation. Moreover, the increases in grid losses and loading factor of the equipment are small and exclusively caused by the desired voltage decrease. Nevertheless, the capacity of WAVC-T to reduce maximum voltage deviations falls in voltage-inhomogeneous grids.

Inverters' Reactive Power Support (IRPS) has a significant effect on the voltage at the end of MVfeeders, where maximum voltage deviations normally appear. Moreover, the additional energy losses caused by this method are limited. However, voltage deviation reductions are not sufficient in any of the studied grids.

The combination of WAVC-T and IRPS has demonstrated to be able to manage voltage deviations completely in all of the analysed grids. One remarkable effect of implementing IRPS in addition to WAVC-T is an observed improvement of the voltage inhomogeneity in all the studied MV-grids. As inhomogeneity is main burden for the proper operation of WAVC-T, the simultaneous implementation of both methods has to be prioritised. Further investigations have to be carried out in order to reach a firm conclusion of the behaviour of IRPS regarding voltage inhomogeneity in MV-grids. Another aspect that has to be investigated is the effect of both methods on the number of tap switchings and consequently on the maintenance and lifetime of the OnLoad Tap-Changer (OLTC).

\section{References}

[1] German Federal Ministry of Economics and Technology (BMWi), "First Monitoring Report: Energy of the future", December 2012.

[2] "European Standard EN 50160: Voltage characteristics of electricity supplied by public distribution networks", February 2011.

[3] Deutsche "Anergie-Agentur, "Ausbau- und Innovationsbedarf der Stromverteilnetze in Deutschland bis 2030", December 2012.

[4] H. Gremmel, "ABB Switchgear Manual”, Edition 11, Cornelsen Lehrbuch, December 1999.

[5] B. Gwisdorf, T. Borchard, T. Hammerschmidt, C. Rehtanz, "Technical and economic evaluation of voltage regulation strategies for distribution grids with a high amount of fluctuating dispersed generation units", IEEE, September 2010.

[6] Verband der Elektrotechnik, Elektronik und Informationstechnik,

"VDE-AR-N4105: Erzeugungsanlagen am Niederspannungsnetz: Technische Mindestanforderungen für Anschluss und Parallelbetrieb von Erzeugungsanlagen am Niederspannungsnetz", August 2011.

[7] Bundesverband der Energie- und Wasserwirtschaft, "Richtlinien für Anschluss und Parallelbetrieb von Erzeugungsanlagen am Mittelspannungsnetz“, June 2008.

[8] Westnetz Gmbh: „Technische Anschlussbedingungen Mittelspannung“, January 2013. 